\title{
Three-Mode Vortex Wave Generator with Double-Layer Patch Antennas
}

\author{
Jun Hu (D), Tong-Tong Qiu, Xue Lan, Wei Xu, and Song-Song Qian \\ Ministerial Key Laboratory of JGMT, Nanjing University of Science and Technology, Nanjing, China \\ Correspondence should be addressed to Jun Hu; junhu@njust.edu.cn
}

Received 27 December 2018; Revised 22 February 2019; Accepted 31 March 2019; Published 2 May 2019

Academic Editor: Shiwen Yang

Copyright (C) 2019 Jun Hu et al. This is an open access article distributed under the Creative Commons Attribution License, which permits unrestricted use, distribution, and reproduction in any medium, provided the original work is properly cited.

With the development of Multiple-Input Multiple-Output technologies, researches on radio vortex wave with orbital angular momentum have become active. At the same time, generators of miniaturized planar antennas have become very interesting. Considering multilayer package, we proposed a uniform circle array consisting of four double-layer patch antennas with microstrip backfeeds. The antenna can generate three modes of vortex waves, which works at frequency $7.6 \mathrm{GHz}$ with a bandwidth $8.5 \%$. Compared with orbital angular momentum antennas of phased uniform circular array with other cells, this proposed structure can be easily integrated with planar feeding networks and decoupling networks.

\section{Introduction}

Vortex waves and Multiple-Input Multiple-Output (MIMO) systems are two different topics. MIMO and massive MIMO are keys of the $5 \mathrm{G}$ communication systems and have been widely discussed over the past decades. Compared to MIMO, vortex waves with orbital angular momentum (OAM) in the light spectrum have been relatively old, and the researches on them in the radio spectrum are newly active. Some specified MIMO systems and radio vortex waves result in the same Eigen-mode [1]. Among all MIMO systems, we believe that radio vortex wave generators have the following advantages. The first is on the beam. Its beam is a Bessel beam with an angular helical orthogonal spiral [2]. The second is on the modulation. Its modulated generation signals of vortex waves are based on Fourier transform, including those in uniform circle arrays (UCA) [3, 4], circle wave-guides [5], reflectivebased generators, and lens-based generators. The third is on the imaging. Its OAM-based imaging method has a higher angular resolution.

We have investigated the recently works on radio OAM. The OAM modes of radio vortex waves from UCA were researched from a theoretical aspect [6], where factors affecting the mode distribution were analyzed. Later, generators carrying plane spiral orbital angular momentum (PSOAM) were proposed to realize the beam control of OAM and to evolve on the shape of UCA [7]. Four-dimensional antenna arrays were designed to broaden the prospect of OAM waves [8], which realized multiple orbital angular momentum modes. Combined with metasurface, dual-mode dual-polarization OAM based on transmissive metasurface was designed [9]. The mode multiplexing of OAM in MIMO systems is also studied [10]. From the investigation, we find that the generation of radio vortex waves with OAM has the following difficulties: how to reduce the size of generators, how to alleviate mutual-coupling among elements, how to enhance bandwidths and scopes, and how to make packages with low profiles.

This paper focuses on the vortex wave generator with low profiles and investigates the mutual-coupling effects by modifying the radius of arrays. We will propose a three-mode vortex wave generator with double-layer patch antennas. The proposed method facilitates the package of the OAM antenna, promotes the integration with decoupling networks, and advances the designs of feeding networks on different layers.

\section{Antenna Designing}

2.1. Basic Formulas of UCA. As in [11], the electric field with orbital angular momentum has the following terms:

$$
\vec{E}_{\mathrm{OAM}}^{\alpha}(\vec{r})=\vec{E}(z, \rho) e^{j \alpha \phi}
$$




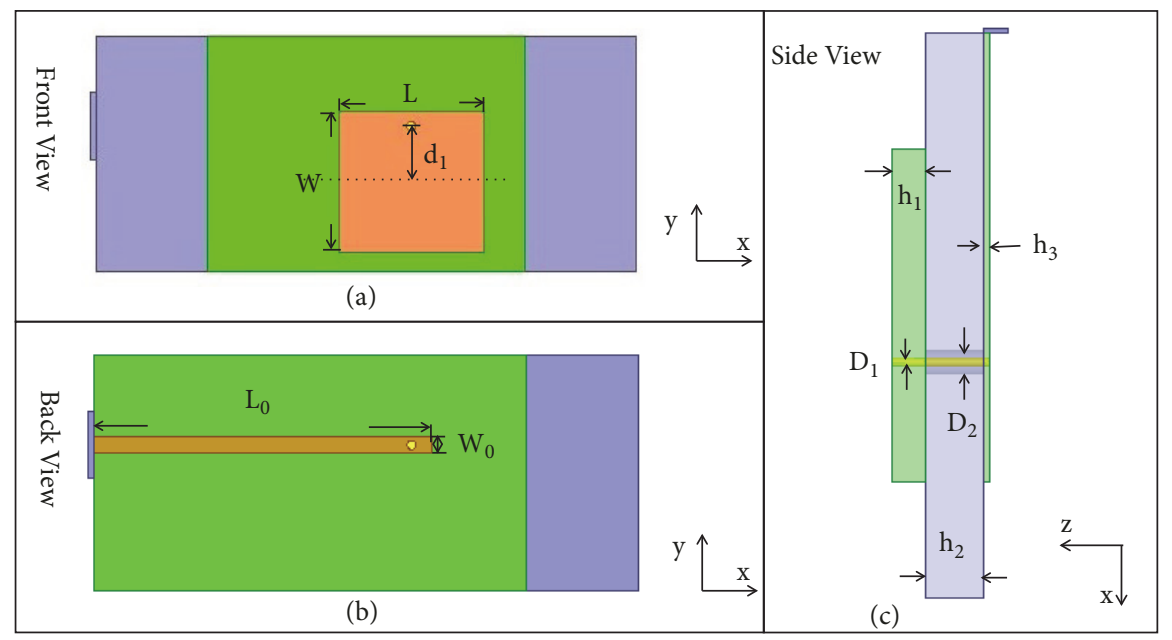

FiguRE 1: Schematic diagram of the cell antenna.

where $\vec{E}(z, \rho)$ denotes the Bessel beam, $\alpha$ denotes the topological index, and $(z, \rho, \phi)$ denotes the coordinate in the cylinder system. By an N-element phased array with a DFTbased modulation, $\vec{E}(z, \rho)$ can be determined in the UCA. The theories of the UCA consisting of point sources have been built, but the designs of the UCA consisting of planar antennas should be investigated carefully. Considering no mutual-coupling effects among elements, we can obtain the left term in (1) as follows:

$$
\vec{E}_{\mathrm{OAM}}^{\alpha, N}(\vec{r})=\vec{E}_{\text {Cell }}(\vec{r}) F_{\mathrm{OAM}}^{\alpha, N}(\vec{r})
$$

where

$$
\begin{aligned}
F_{\mathrm{OAM}}^{\alpha, N}(\vec{r}) & =\sum_{n=1}^{N} \frac{1}{\left|\vec{r}-\vec{r}_{n}\right|} e^{-j k\left|\vec{r}-\vec{r}_{n}\right|} e^{j \alpha \phi_{n}} \\
\vec{r}_{n} & =\left(a \sin \phi_{n}, a \cos \phi_{n}, 0\right) \\
\phi_{n} & =\frac{2 \pi n}{N}, \quad n=1,2, \ldots, N
\end{aligned}
$$

and $\vec{E}_{\text {Cell }}$ denotes the primary pattern of patch, where $a$ denotes the radius of the circle array.

2.2. Multilayer Patch OAM. Although vortex waves are mainly determined by the array factor, the qualities of vortex waves are affected by primary pattern and mutual-coupling effects. In order to design a generator which can be easily packaged and has great isolations, we select a double-layer patch antenna [10] as elements.

The antenna is a double-layer patch antenna as shown in Figure 1. It consists of two substrate layers with ground in the middle. The configuration and parameters are shown in Figure 1 and Table 1. The top layer supports a rectangle radiation patch as shown in Figure 1(a) and the bottom layer supports the microstrip feed line as shown in Figure 1(b). A via goes from the feed line to the radiation patch through the
TABLE 1: Parameters of the generator (unit: $\mathrm{mm}$ ).

\begin{tabular}{lccc}
\hline parameters & value & parameters & value \\
\hline$W$ & 12.2 & $\mathrm{~d}_{1}$ & 5 \\
$L$ & 13 & $\mathrm{~d}_{2}$ & 26 \\
$\mathrm{~W}_{0}$ & 1.5 & $\mathrm{~h}_{1}$ & 3 \\
$\mathrm{~L}_{0}$ & 30.1 & $\mathrm{~h}_{2}$ & 5 \\
$\mathrm{~W}_{1}$ & 46 & $\mathrm{~h}_{3}$ & 0.5 \\
$\mathrm{~L}_{1}$ & 46.4 & $\mathrm{D}_{1}$ & 2 \\
$\mathrm{~L}_{2}$ & 66.4 & $\mathrm{D}_{2}$ & 0.8 \\
\hline
\end{tabular}

layers and the ground as shown in Figure 1(c), which incidents the radiation magnetic slots.

The proposed vortex wave generator is shown in Figure 2. Its four radiation patches are arranged in Figure 2(a), and their feeding strips are arranged in Figure 2(b). Parameters are shown in Table 1. Compared with the reported patch OAM-antennas, the radiation areas of them are not reduced. As the OAM antenna designed in [11], most types of the patch OAM-antennas have CPW feedings, where the effective radiation areas of the patch are reduced. In the proposed antenna, chips are powered by probes which are converted into microstrip feed at the bottom layer. Another advantage is that the impedance matching is convenient. At the same time, the ground plane is the middle slice of the sandwich structure. This can make the proposed structure packaged with low profiles and can make decoupling networks attached conveniently [12].

\section{Simulation and Performance}

In the previous section, we introduced the schematic of the proposed double-layer generator. In this section, we discussed its performances. These discusses are focused on the mutual-coupling effects for different radius of UCA.

First, we investigated the performance of the single antenna. As before, the length is $13 \mathrm{~mm}$, the width is $12.2 \mathrm{~mm}$, the thickness of the top layer is $3 \mathrm{~mm}$, and the thickness of the 


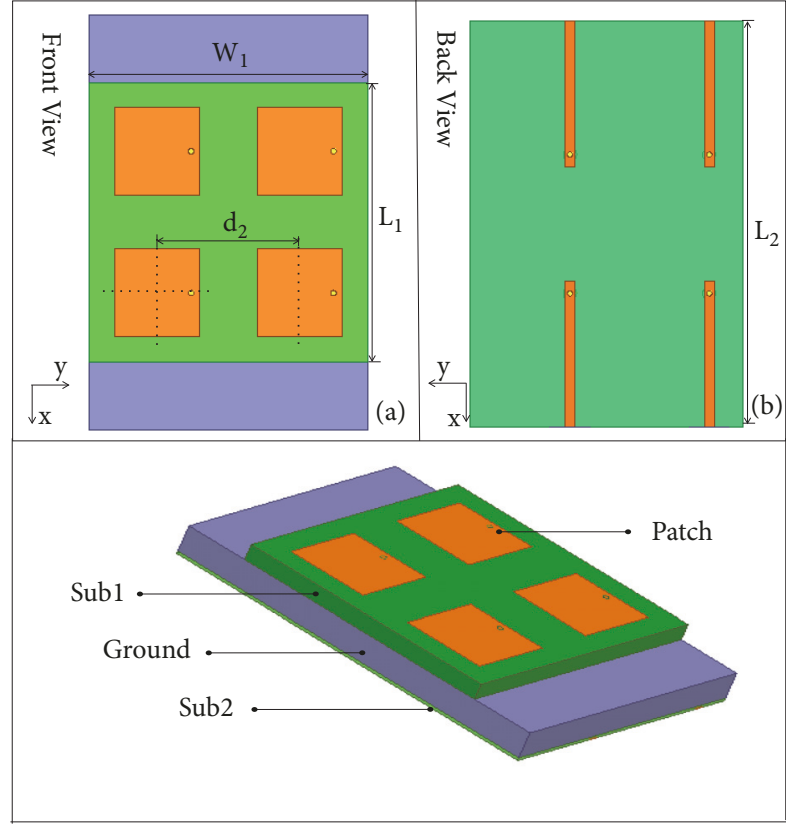

FIgURE 2: The configuration of the generator.

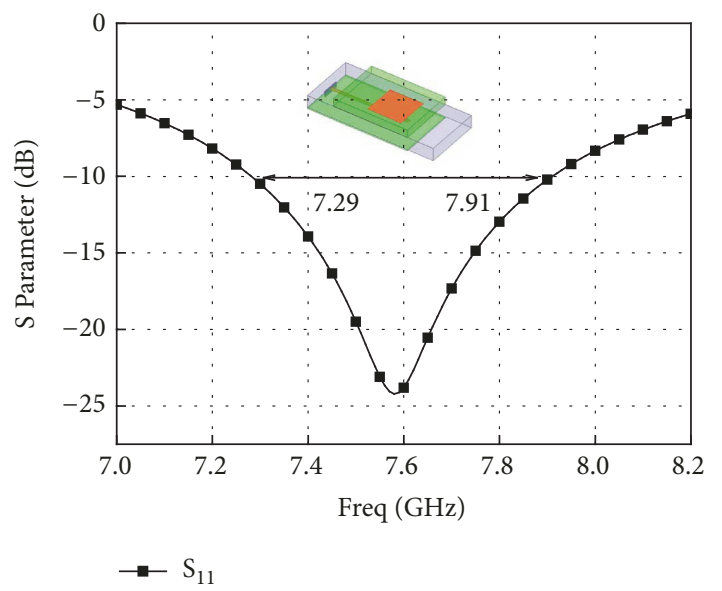

FIgURE 3: Return loss of the cell antenna.

bottom layer is $0.5 \mathrm{~mm}$. The substrates of different layers are the same, whose permittivity is $\varepsilon_{r}=2.2$. The performances are shown in Figure 3. Figure 3 is on the return loss of a single antenna, which suggests that the antenna works at $7.6 \mathrm{GHz}$ with its bandwidth below $-10 \mathrm{~dB}$ which is $8.2 \%$.

Second, we discussed the performance of the UCA. Since the goal is to obtain a generator of small size, we attempted to find the optimized radius. Take the distance between the elements of $26 \mathrm{~mm}$ while the radius of the entire circular array is $18.3 \mathrm{~mm}$, about $0.46 \lambda_{0}$. The array has ports where four signals with different phase-delays are attached. Results are shown in Figures 4-6. Figure 4 shows the augments and phases of vortex waves with different topological index of modal -1 , modal 0 , and modal 1 . It suggests that three-mode vortex waves can be obtained. Figure 5 shows the return loss and inserting loss for the OAM generator, which suggests that

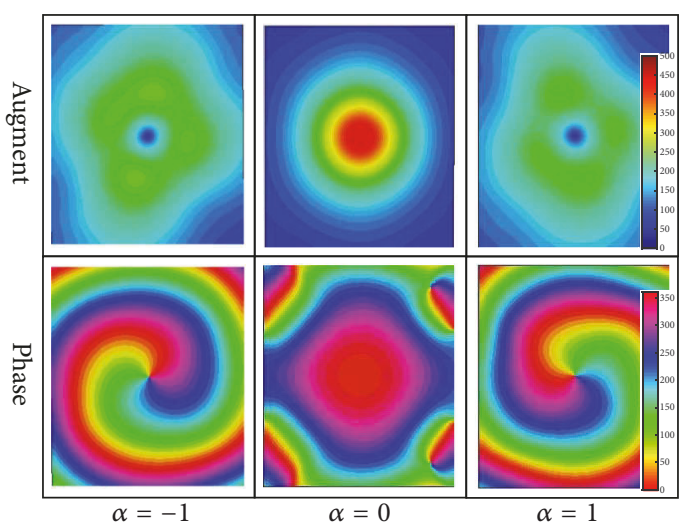

FIGURE 4: Augments and phases of vortex waves with different topological index of modal-1, modal 0 , and modal $1(\mathrm{a}=18.3 \mathrm{~mm})$.

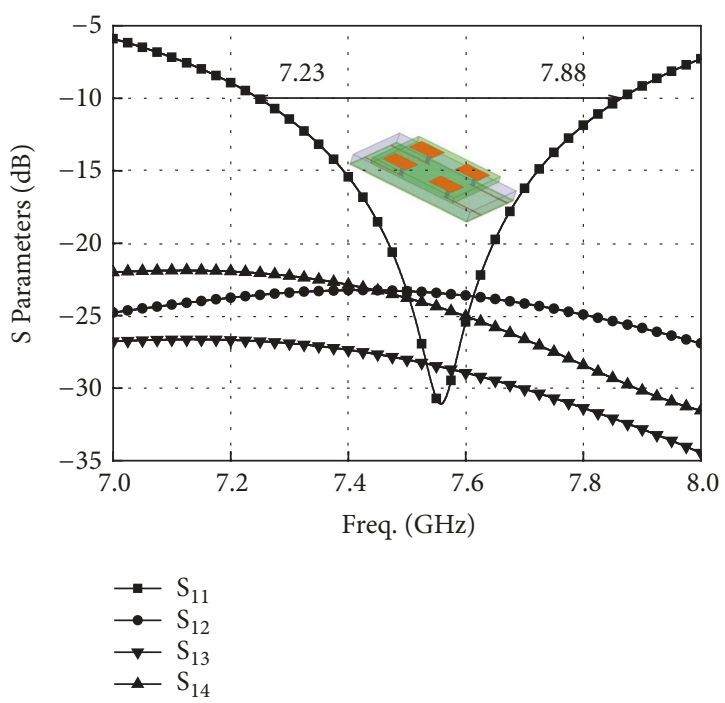

FIGURE 5: Return loss and inserting loss for the OAM generator $\left(\mathrm{a}=18.3 \mathrm{~mm}, 0.46 \lambda_{0}\right)$.

the bandwidth of return loss below $-10 \mathrm{~dB}$ is $8.5 \%$ and the level of the mutual-coupling is lower than $-20 \mathrm{~dB}$.

We tried to reduce the radius of the OAM generator to test the limits of the array. Since the radius was less than $14.8 \mathrm{~mm}$, the $S$ parameters were obviously changed. The distance was reduced by $14.8 \mathrm{~mm}$ and the antenna was made simulations. Results are shown in Table 2 and Figures 6-9. Figure 6 shows simulated patterns of generator with different radiuses of array; some details of patterns are discussed in Table 2 . When the radius becomes smaller, the gain is reduced while the main lobe direction changes and the angular width with $3 \mathrm{~dB}$ becomes larger. Since the transmission distance of the OAM wave is determined by the main lobe direction of the vortex beam offsets, it indicates that the radius of the circular array should be large enough to increase the transmission distance of the wave. Figure 7 shows the return loss and inserting loss for the OAM generator. It suggests that the bandwidth of return loss below $-10 \mathrm{~dB}$ is $3.5 \%$ and the level of mutualcoupling is about $-17 \mathrm{~dB}$. Figure 8 shows the surface current 


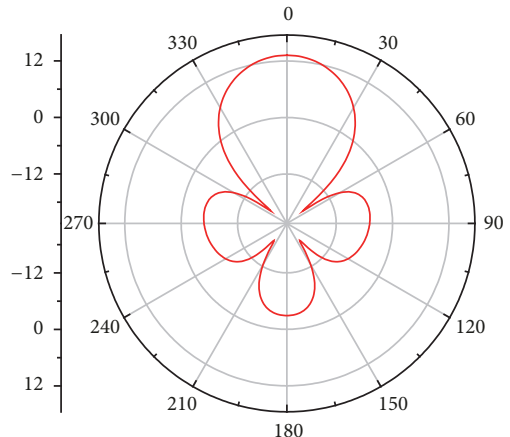

(a) $\mathrm{a}=18.3 \mathrm{~mm}$; mode 0

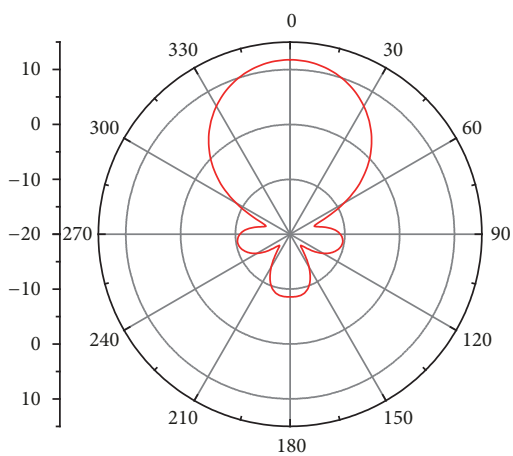

(c) $\mathrm{a}=14.8 \mathrm{~mm}$; mode 0

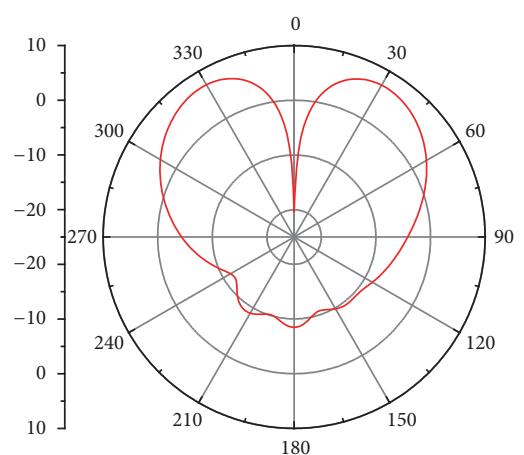

(b) a=18.3mm; mode 1, -1

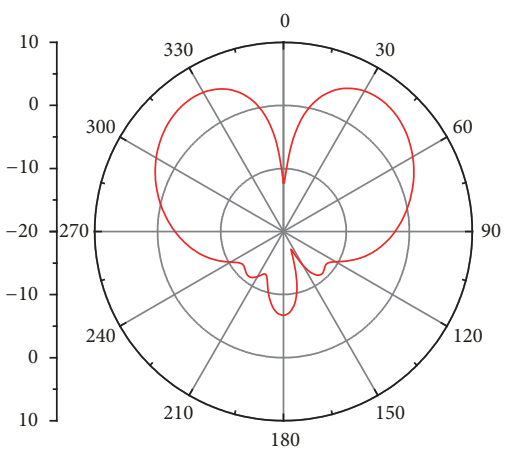

(d) a=14.8mm; mode $1,-1$

FIGURE 6: Simulated patterns of generator with different radiuses of array.

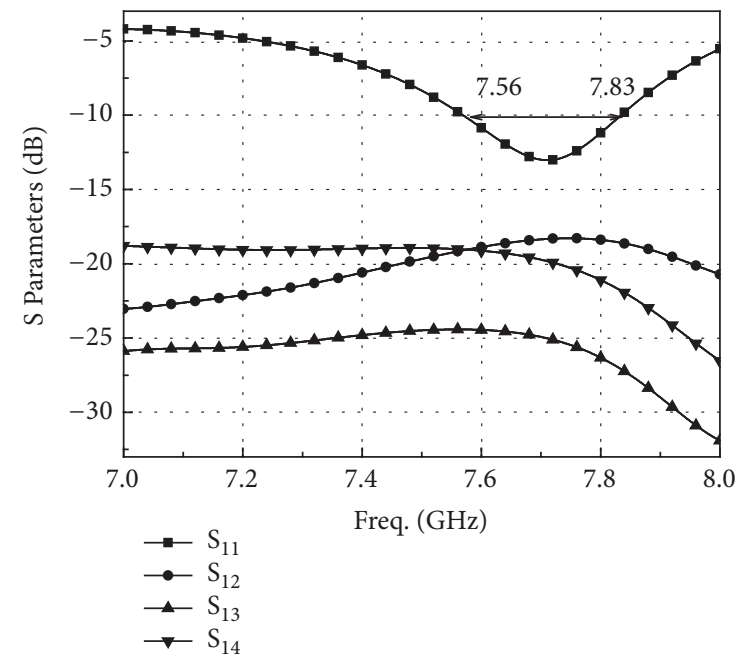

FIGURE 7: Return loss and inserting loss for the OAM generator $(\mathrm{a}=$ $14.8 \mathrm{~mm})$.

of the generator of different radiuses. As can be seen in this picture, when the radius is $18.3 \mathrm{~mm}$, different patches are well isolated at frequency $7.6 \mathrm{GHz}$. When the radius falls to $14.8 \mathrm{~mm}$, currents on different patches begin to interact. At the same time, the current intensity becomes lower. Figure 9 shows that three-mode vortex waves are still obtained when the radius is $14.8 \mathrm{~mm}$. However, the dark area of the vortex becomes smaller. So from the perspective of the vortex,
TABLE 2: Parameters of patterns.

\begin{tabular}{lcc}
\hline \multirow{2}{*}{ Items } & \multicolumn{2}{c}{ Performances } \\
\hline Gain & $\mathrm{a}=18.3 \mathrm{~mm}$ & $\mathrm{a}=14.8 \mathrm{~mm}$ \\
Main lobe direction & $7.2 \mathrm{dBi}$ & $6.0 \mathrm{dBi}$ \\
Angular with 3dB & $32 \mathrm{deg}$ & $37 \mathrm{deg}$ \\
Side lobe level & $40.8 \mathrm{deg}$ & $46.6 \mathrm{deg}$ \\
Total efficiency & $-15.7 \mathrm{~dB}$ & $-12.7 \mathrm{~dB}$ \\
\hline
\end{tabular}

the radius tries to be small. But if the radius is too small, decoupling is needed.

Investigating multiple parameters, we confirm the current optimal array radius. First, the radius cannot be too large. The large radius can bring with side-lobes and low radiation efficiency as in Table 2. In some cases, the small radius is appropriate. Second, the distance between the antenna elements cannot be too small. As shown in Table 2 and Figures 7 and 8, if the radius is too small, the level mutualcoupling between array elements will be severe. At the same time, the bandwidth and the gain will be weak. According to the above, we choose $18.3 \mathrm{~mm}$ as a relatively suitable radius.

At this point, the size of the circular array is much smaller than that of similar UCA [9]. It also has a higher bandwidth and lower mutual-coupling than antennas of the same resonant frequency.

Figures 10 and 11 show the manufactured OAM antenna and measured results, respectively. Because of the consistency 


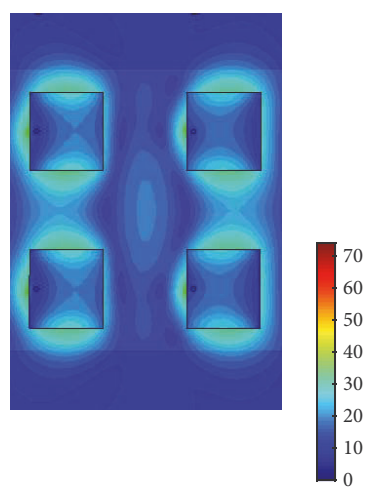

(a) $\mathrm{a}=18.3 \mathrm{~mm}$

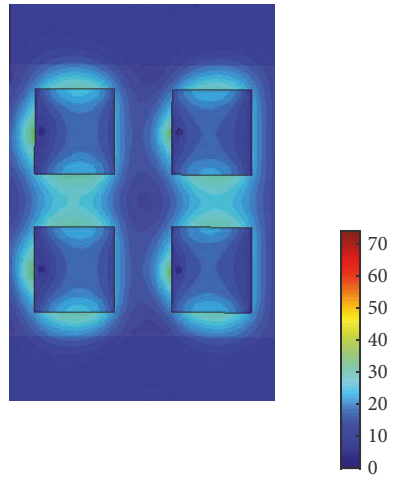

(b) $\mathrm{a}=14.8 \mathrm{~mm}$

FIGURE 8: Surface current of the generator with different radiuses (unit: A/m(log)).

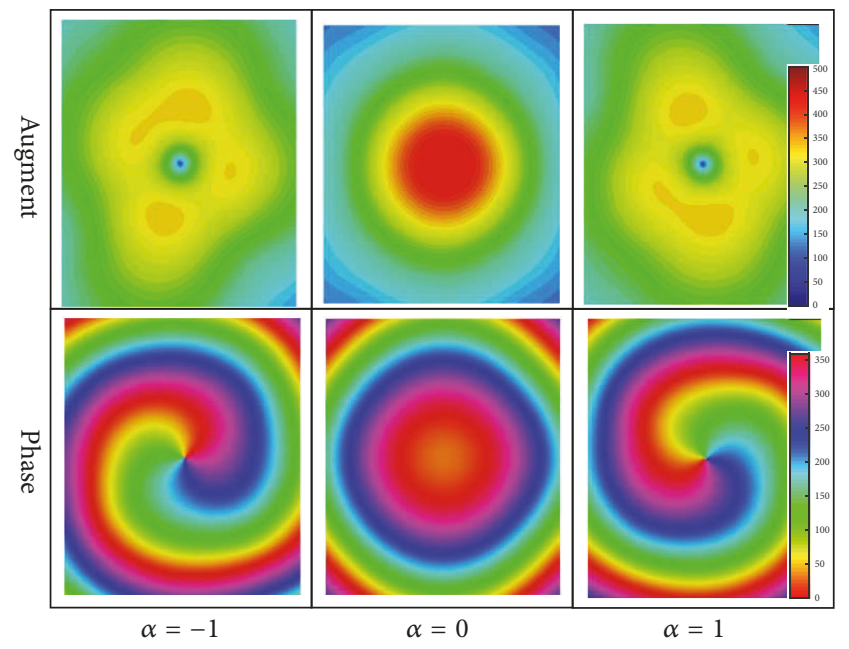

FIGURE 9: Augments and phases of vortex waves with different topological index of modal-1, modal 0 , and modal $1(\mathrm{a}=14.8 \mathrm{~mm})$.

of each port, we have selected four typical S-parameter curves. The reflection coefficient of elements and the mutualcoupling coefficients between them can be seen from Figure 11. The simulated bandwidth and measured bandwidth agree with each other well, and the measured bandwidth is slightly shifted towards the lower frequency. Meanwhile, elements are well isolated with each other. The difference between simulated results and measured results may come from the permittivity of substrate and the effect of manual assembly. Figure 12 shows the simulated and measured radiation patterns of this generator. As shown in the plots, simulated and measured radiation patterns show a reasonable agreement with each other.

According to the above discussions, we found that the proposed method can be used to build a three-mode generator of vortex waves, and its performances are mainly determined by the radius of the array. For reduced array, the vortex waves can be generated in a reduced scope, and its return losses and insert losses deteriorate.

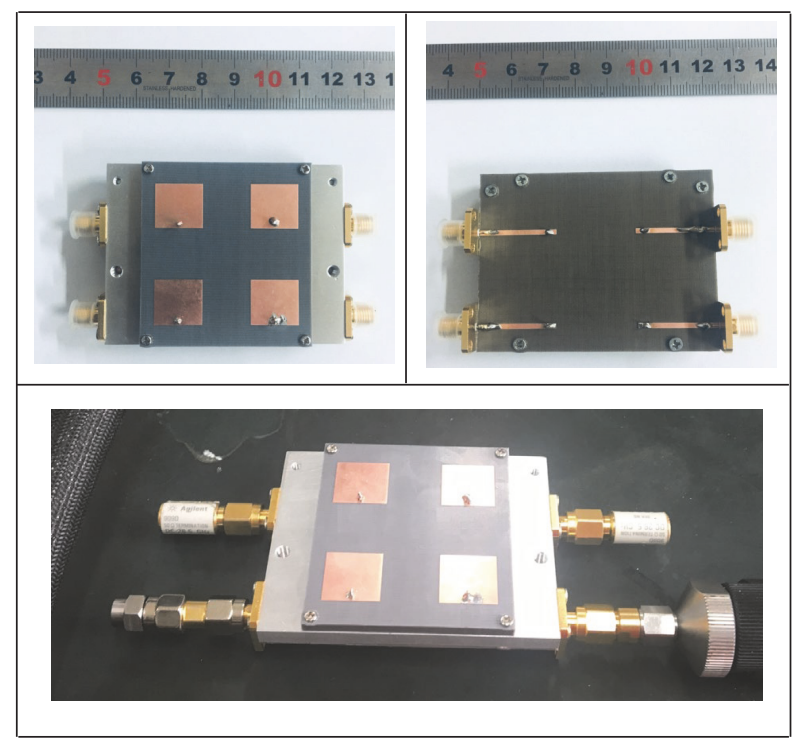

FIGURE 10: Antenna prototype and testing environment.

\section{Conclusion}

In this paper, we have proposed a three-mode vortex wave generator of 4-element double-layer planar antenna array. The generator is designed at frequency $7.6 \mathrm{GHz}$. Although the vortex waves would be obtained for different array sizes, their bandwidths and gains are different. With the increase of array radius from $14.8 \mathrm{~mm}$ to $18.3 \mathrm{~mm}$, the bandwidth of the return loss below $-10 \mathrm{~dB}$ changes from $3.5 \%$ to $8.6 \%$, and the gain of generator changes from $6.0 \mathrm{dBi}$ to $7.2 \mathrm{dBi}$. The doublelayer circular array is of low profile, and its thickness is $8.5 \mathrm{~mm}$. Contributed to the planar antennas and their planar feedings, the generator can be packaged into an integrated OAM generator. For sandwich structure with ground in the middle slice, the proposed structure can be integrated with decoupling networks for a reduced size. 


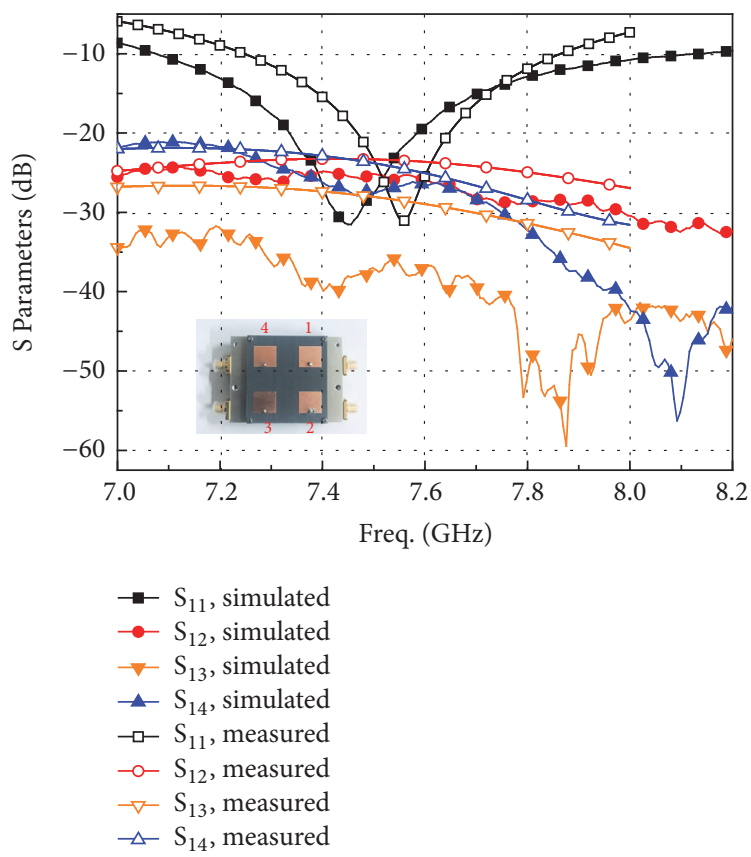

FIGURE 11: Simulated and measured return loss for the OAM generator $(\mathrm{a}=18.3 \mathrm{~mm})$.
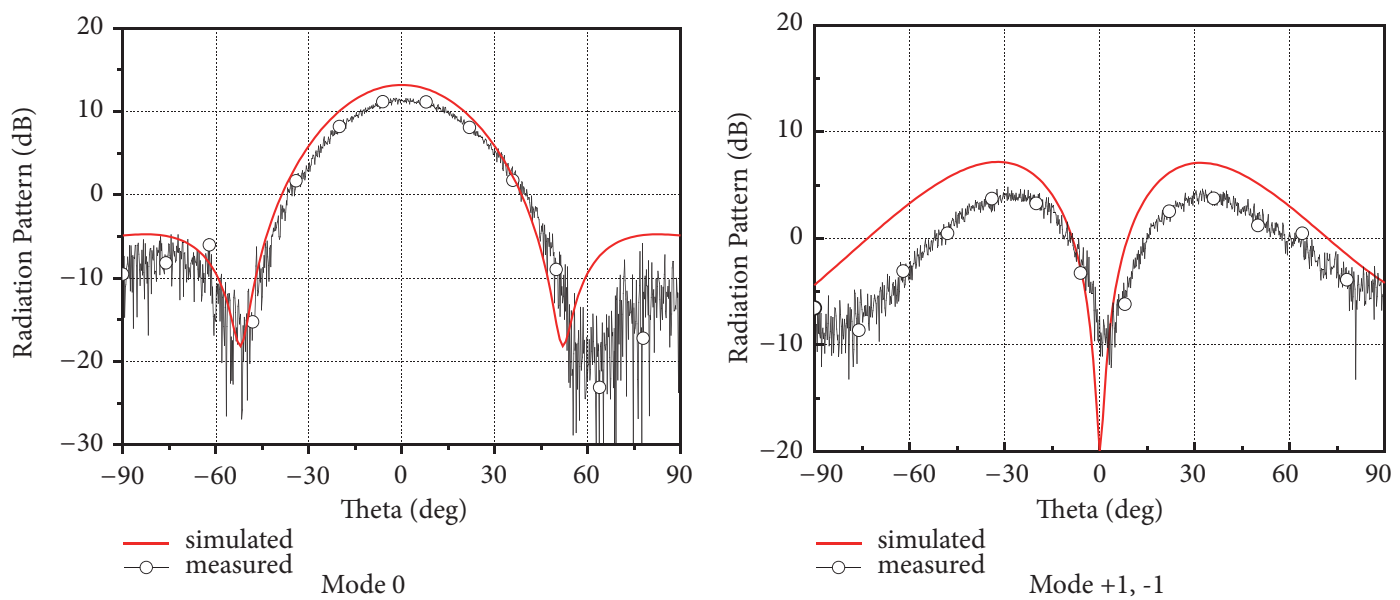

FIGURE 12: Simulated and measured patterns.

\section{Data Availability}

The data used to support the findings of this study are included within the article.

\section{Conflicts of Interest}

The authors declare that there are no conflicts of interest regarding the publication of this paper.

\section{Acknowledgments}

This work was supported by the National Natural Science Foundation under Grant 61901019.

\section{References}

[1] O. Edfors and A. J. Johansson, "Is orbital angular momentum (OAM) based radio communication an unexploited area?" IEEE Transactions on Antennas and Propagation, vol. 60, no. 2, pp. 1126-1131, 2012.

[2] A. M. Yao and M. J. Padgett, "Orbital angular momentum: origins, behavior and applications," Advances in Optics and Photonics, vol. 3, no. 2, pp. 161-204, 2011.

[3] L. Torner, J. P. Torres, and S. Carrasco, "Digital spiral imaging," Optics Express, vol. 13, no. 3, pp. 873-881, 2005.

[4] B. Thidé, H. Then, J. Sjöholm et al., "Utilization of photon orbital angular momentum in the low-frequency radio domain," Physical Review Letters, vol. 99, no. 8, Article ID 087701, 2007. 
[5] S. Zheng, Y. Chen, Z. Zhang et al., "Realization of beam steering based on plane spiral orbital angular momentum wave," IEEE Transactions on Antennas and Propagation, vol. 66, no. 3, pp. 1352-1358, 2018.

[6] T. Yuan, Y. Cheng, H. Wang, and Y. Qin, "Mode characteristics of vortical radio wave generated by circular phased array: theoretical and experimental results," IEEE Transactions on Antennas and Propagation, vol. 65, no. 2, pp. 688-695, 2017.

[7] S. Zheng, W. Zhang, Z. Zhang, X. Jin, H. Chi, and X. Zhang, "Generation and propagation characteristics of electromagnetic vortices in radio frequency," Photonics Research, vol. 4, no. 5, pp. B9-B13, 2016.

[8] C. Sun, S. Yang, Y. Chen, J. Guo, and S. Qu, "Realization of multiple orbital angular momentum modes simultaneously through four-dimensional antenna arrays," Scientific Reports, vol. 8, no. 149, 2018.

[9] X. Qi, Z. Zhang, X. Zong, X. Que, Z. Nie, and J. Hu, “Generating dual-mode dual-polarization oam based on transmissive metasurface," Scientific Reports, vol. 9, no. 1, 2019.

[10] R.-L. Xia, S.-W. Qu, P.-F. Li, Q. Jiang, and Z.-P. Nie, "An efficient decoupling feeding network for microstrip antenna array," IEEE Antennas and Wireless Propagation Letters, vol. 14, pp. 871-874, 2015.

[11] Q. Bai, A. Tennant, and B. Allen, "Generation of orbital angular momentum (OAM) radio beams with phased patch array," in Proceedings of the Antennas and Propagation Conference, vol. 9, pp. 410-413, 2014.

[12] K. K. Mojtaba, R. H. Hamid, and M. A. N. Sajad, "Wide scan phased array patch antenna with mutual coupling reduction," IET Microwaves, Antennas \& Propagation, vol. 12, no. 12, pp. 1932-1938, 2018. 


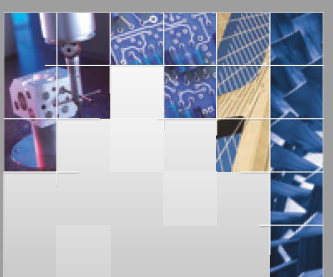

\section{Enfincering}
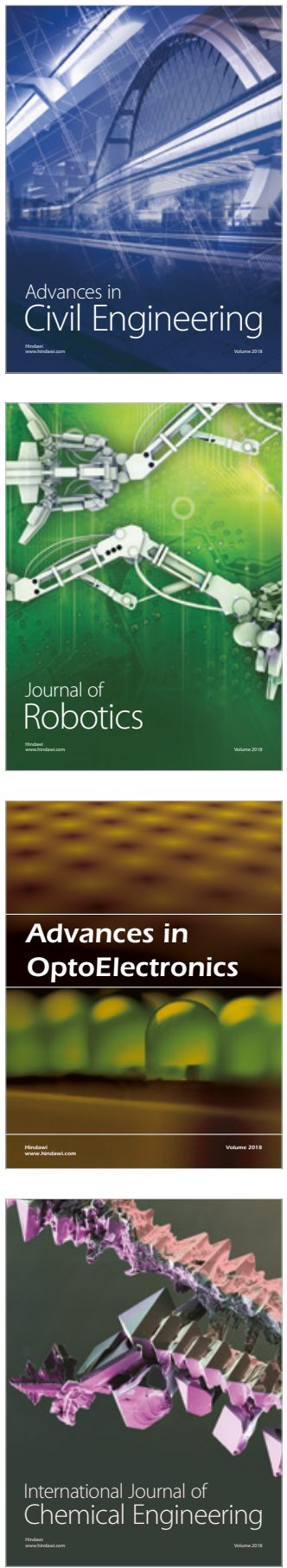

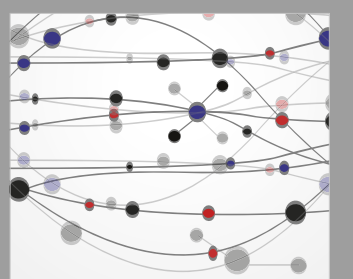

\section{Rotating \\ Machinery}

The Scientific World Journal

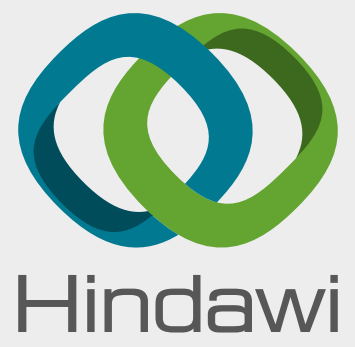

Submit your manuscripts at

www.hindawi.com
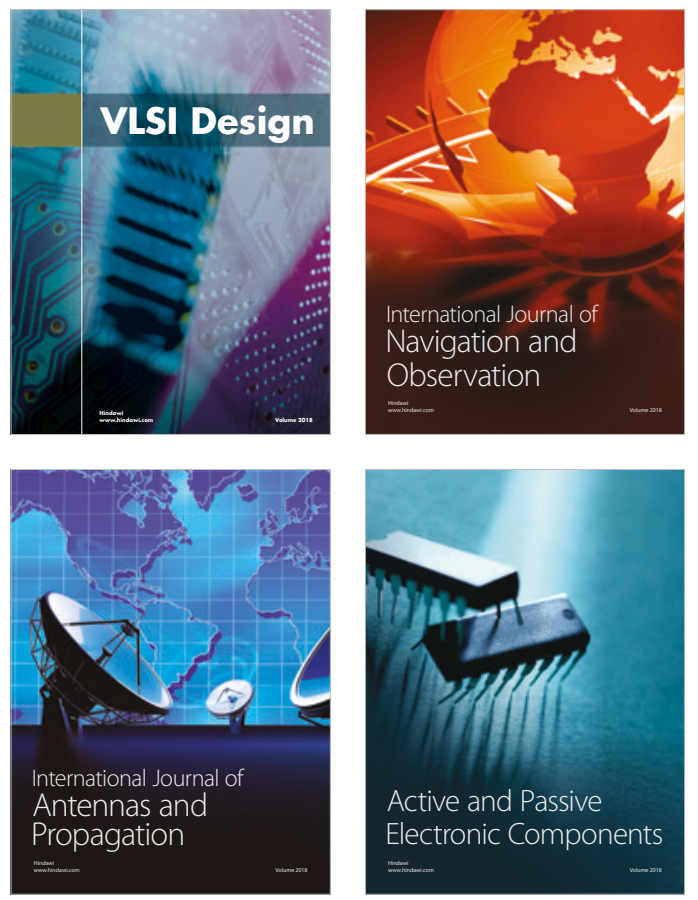
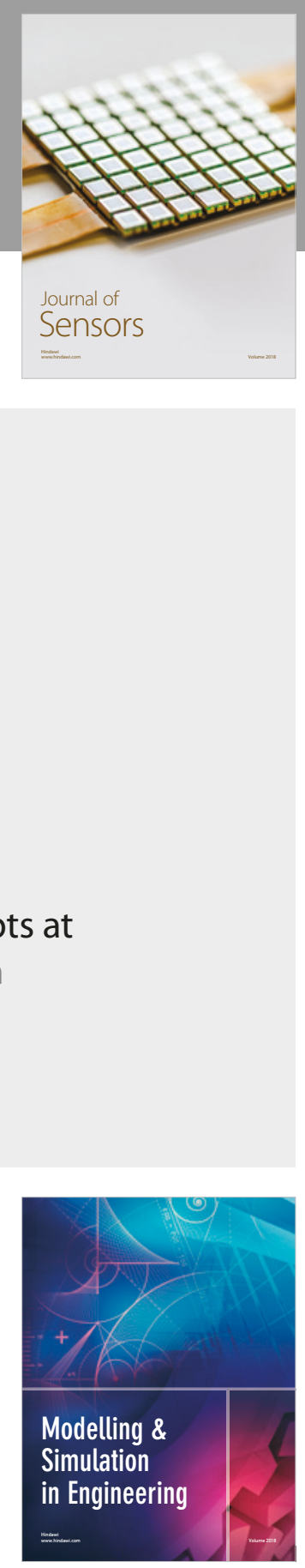

\section{Advances \\ Multimedia}
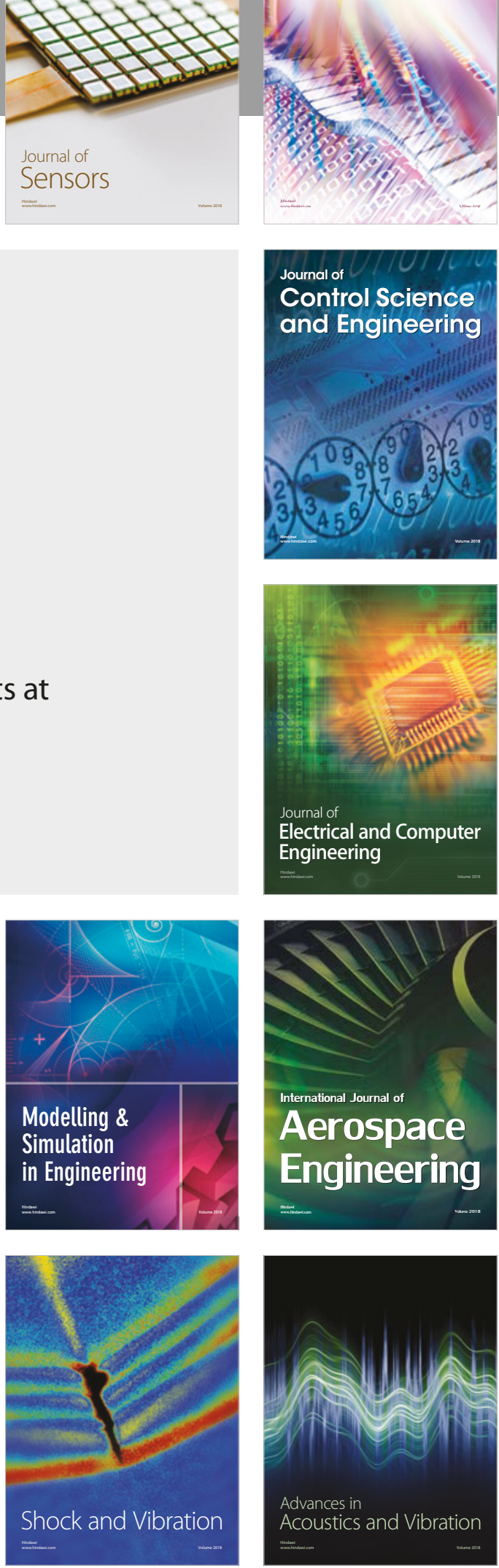